\title{
Natural course of myopic traction maculopathy and factors influencing progression and visual acuity
}

\author{
Shiwei Li, Tingting Li, Xiangning Wang, Xuan Cai, Bin Lu, Yan Chen, Chang Liu and Qiang Wu* (D)
}

\begin{abstract}
Background: To describe the natural course of myopic traction maculopathy (MTM) and determine predictive factors for its progression and visual prognosis.

Methods: This retrospective observational study included 113 MTM patients (113 eyes). Best-corrected visual acuity (BCVA) measurements and optical coherence tomography findings were recorded.

Results: Over a mean follow-up of $38.2 \pm 11.1$ months, 49 of 113 eyes (43.4\%) progressed. The progression rate of outer schisis prominently located in the fovea or posterior staphyloma was significantly higher than that of outer schisis prominently located in paravascular areas $(P=0.0011)$. MTM with partial posterior vitreous detachment during the follow-up progressed more rapidly than MTM without $(P=0.0447)$. Patients with older age (> 65 years), without domed-shaped macula and with defects in the ellipsoid zone (EZ) had worse BCVA at the last visit $(P=0.0416, P=0.0494$ and $P=0.0130)$. Multiple linear regression analysis showed that $B C V A$ and defects in the EZ at baseline were significantly associated with the final BCVA $(P<0.0001$ and $P=<0.0001$, respectively).
\end{abstract}

Conclusions: MTM has a high possibility for progression. Outer schisis located predominantly in the fovea or posterior staphyloma or with partial posterior vitreous detachment exhibits rapid progression. The integrity of the EZ is related to visual prognosis.

Keywords: High myopia, Myopic traction maculopathy, Natural course, Visual acuity, Optical coherence tomography

\footnotetext{
* Correspondence: qiang.wu@shsmu.edu.cn

Department of Ophthalmology, Shanghai Jiaotong University Affiliated Sixth

People's Hospital, No. 600 Yishan Rd, 200233 Shanghai, China
}

(C) The Author(s). 2021 Open Access This article is licensed under a Creative Commons Attribution 4.0 International License, which permits use, sharing, adaptation, distribution and reproduction in any medium or format, as long as you give appropriate credit to the original author(s) and the source, provide a link to the Creative Commons licence, and indicate if changes were made. The images or other third party material in this article are included in the article's Creative Commons licence, unless indicated otherwise in a credit line to the material. If material is not included in the article's Creative Commons licence and your intended use is not permitted by statutory regulation or exceeds the permitted use, you will need to obtain permission directly from the copyright holder. To view a copy of this licence, visit http://creativecommons.org/licenses/by/4.0/. The Creative Commons Public Domain Dedication waiver (http://creativecommons.org/publicdomain/zero/1.0/) applies to the data made available in this article, unless otherwise stated in a credit line to the data. 


\section{Background}

Myopic traction maculopathy (MTM) refers to a series of pathological changes in the macula in high myopia, including vitreomacular traction, an epimacular membrane, retinoschisis, a lamellar macular hole (LMH) and foveal detachment [1]. Damage to the outer retinal structure or development to foveal detachment and a full-thickness macular hole in the advanced stage can cause visual impairment in MTM, and surgical intervention is recommended to promote anatomical reattachment of the retina and visual recovery [2-5]. Retinoschisis is a major characteristic lesion of MTM, and the incidence of retinoschisis in high myopia with posterior staphyloma is as high as $31.3 \%$ [6]. Retinoschisis can occur in the fovea and extrafovea and in different locations of the intraretinal neural layers due to different dominant pathological factors. However, the mechanism of retinoschisis has not yet been fully elucidated, and many studies have shown that inward and tangential forces produced by partial posterior vitreous detachment (PVD), an epiretinal membrane, arteriosclerosis, and a stiff internal limiting membrane (ILM) and outward traction generated by asynchronous global elongation and posterior staphyloma may play important roles in its pathogenesis [7-11].

Recently, several studies have indicated that the progression rate of MTM varies, that paravascular abnormities and paravascular inner retinoschisis may be associated with the pathogenesis of foveoschisis, and that the severity of retinoschisis in MTM can affect its progression regardless of whether cataract surgery is performed [12-17]. Poor baseline visual acuity is often found in entire macula-involved retinoschisis eyes accompanied by a disruption in the ellipsoid zone (EZ) [16], and progression of MTM can lead to worse visual outcomes in its natural course [15]. However, these studies have focused little on identifying the evolution of retinoschisis prominent in different locations caused by different initiation factors, on the role of inner retinoschisis at paravascular arcades and ILM detachment in the progression of MTM, or on the comparison of the baseline factors affecting visual acuity at follow-up in large series.

Thus, we conducted this study to describe the natural course of MTM, to determine the effects of morphological characteristics of retinoschisis by OCT on MTM progression and to assess the risk factors influencing visual prognosis.

\section{Methods}

\section{Patients}

This retrospective study recruited patients with MTM who initially visited the Department of Ophthalmology of the Sixth People's Hospital Affiliated to Shanghai Jiao Tong University from June 2014 to March 2018. The inclusion criteria were as follows: (1) MTM diagnosed by OCT, and (2) highly myopic eyes defined as an axis length $\geq 26 \mathrm{~mm}$ or a spherical equivalent refractive error $\geq-6.00 \mathrm{D}$. The exclusion criteria were as follows: (1) OCT images with poor quality; (2) a fullthickness macular hole at baseline; (3) a history of vitreoretinal surgery, and (4) other ocular diseases such as glaucoma, retinal vascular diseases or myopic, or age-related macular degenerative diseases. The study adhered to the guidelines of the Helsinki Declaration and had the approval of the Ethics Committee of Sixth People's Hospital Affiliated to Shanghai Jiao Tong University, Shanghai, China. The study was registered in the Chinese clinical trial registry (http://www.chictr.org.cn/, Registration number: ChiCTR2000038824). All patients signed written informed consent for participation.

\section{Clinical examinations}

All patients were given a comprehensive ocular examination. Best-corrected visual acuity (BCVA) and refractive error were measured by applying a Snellen chart, and BCVA was then converted to logarithmic minimal angle of resolution (logMAR) units for statistical analysis. Axial length was measured by an IOL-Master, and the presence of posterior staphyloma was observed by Bscan ultrasonography. Spectral domain OCT (SD-OCT) (Heidelberg Engineering, Heidelberg, Germany) was performed on the MTM eyes. The SD-OCT scanning protocol consisted of an A-scan through the center of the macula with a length between $9.2 \mathrm{~mm}$ and $11.5 \mathrm{~mm}$ in the horizontal and vertical direction and 31 B-scans covering an area of $30^{\circ} \times 25.0^{\circ}$ centered on the fovea at an interval of $256 \mu \mathrm{m}$. Two subtypes of retinoschisis were recorded: outer schisis (occurring in the outer plexiform layer) and inner schisis (occurring in the inner plexiform layer and/or ILM detachment). The eyes were classified into 5 groups based on the size and the location of the outer retinoschisis as proposed by Shimada et al. [15]. The central foveal thickness (CFT) was measured and defined as the distance between the hyperreflective band of the ILM and the hyperreflective band of the retinal pigment epithelium through the central fovea and averaging the values measured in the horizontal and vertical A-scans. Then, we determined the most prominent location (upper and lower vascular arcades or their branch vessels, the fovea or the posterior staphyloma) of outer schisis within a diameter of $10 \mathrm{~mm}$ centered on the fovea from OCT images, in which the maximum neural thickness (MNT), defined as the distance between the hyperreflective band of the ILM and the hyperreflective band of the retinal pigment epithelium, was 
measured. Partial PVD, an epimacular membrane, an LMH and the integrity (intact, partially continuous, or absent) of the EZ were examined by OCT. Dome-shaped macula (DSM) is defined as an inward protrusion of the retinal pigment epithelium of the macula $\geq 50 \mu \mathrm{m}$ in the horizontal or vertical section or both by OCT examination [18].The progression of MTM was characterized as the height of outer schisis increasing by $100 \mu \mathrm{m}$, expansion in the extent of outer schisis, or the development of an LMH, FD or a full-thickness macular hole. MTM improvement was termed a reduction in the height or extent of outer schisis unaccompanied with the development of an LMH, FD or a full-thickness macular hole. Cases that did not meet the standard of progression or improvement were treated as stable based on the definition of Shimada et al. [15]. The follow-up time lasted for at least 2 years. BCVA measurements and OCT examinations were performed in all patients at every visit.

\section{Statistical analysis}

Statistical analysis was performed using SAS software version 9.13 (SAS Institute Inc., Chicago, IL). Data are depicted as the mean \pm standard deviation (SD). The one-way analysis of variance was used for the comparison of continuous variables, and the chi-square test was used for the statistical analysis of count data. If the sample size in the group was $<5$, Fisher's exact test was performed. If the data did not conform to a normal distribution, the Kruskal-Wallis test was performed. We determined the factors influencing BCVA at the last visit using multivariate regression analysis. When $P$ was < 0.05 , the difference was considered statistically significant.

\section{Results}

\section{Baseline characteristics}

We enrolled 113 patients (113eyes) with MTM in this study, and the mean follow-up time was $38.2 \pm 11.1$ months. The baseline characteristics of all patients are listed in Table 1.

\section{Changes in morphological characteristics by OCT}

There were $5,23,8,42$ and 35 eyes in stages S0, S1, S2, S3, and S4 of MTM, respectively, at the last visit. In the follow-up period, 49 eyes $(43.4 \%)$ progressed (Figs. 1 and 2), 45 eyes (39.8\%) remained stable (Fig. 3), and 19 eyes (16.8\%) experienced improvement (Fig. 4). A full-thickness macular hole was found in 1 eye, an LMH developed in 3 eyes, and FD developed in 6 eyes (Fig. 5). At the last visit, ILM detachment was disrupted or disappeared in 4 eyes that did not experience progression.
Table 1 Demographics and baseline characteristics of patients with myopic traction maculopathy

\begin{tabular}{ll}
\hline Variable & Value \\
\hline No. of subjects & 113 \\
No. of eyes & 113 \\
Sex, male/female & $38 / 75$ \\
Age (years),mean \pm SD (range) & $65.1 \pm 9.6$ (34 to 83) \\
Refractive error (D), mean \pm SD & $-12.6 \pm 4.8(-6.0$ to -23.0$)$ \\
(range) & \\
(phakic eyes, $n=66)$ & \\
Axial length (mm), mean \pm SD (range) & $29.3 \pm 1.83(26.00$ to 36.03) \\
BCVA in Snellen equivalent & $20 / 50 \pm 20 / 50(20 / 1000-20 / 20)$ \\
BCVA in logMAR, mean \pm SD & $0.42 \pm 0.39$ \\
Posterior staphyloma (n) & 88 \\
Pseudophakic eyes(n) & 47 \\
Follow-up duration (months) & $38.2 \pm 11.1(24$ to 71$)$ \\
Mean \pm SD (range) & \\
Schisis group (n) & \\
S0 & 8 \\
S1 & 24 \\
S2 & 9 \\
S3 & 46 \\
S4 & 26
\end{tabular}

The most prominent location of the outer schisis $(n)^{a}$

$\begin{array}{ll}\text { Paravascuslar } & 43 \\ \text { Fovea or posterior staphyloma } & 62 \\ \text { Schisis subtype }(n)^{\text {a }} & \\ \text { Outer schisis } & 67 \\ \text { Outer and inner schisis } & 38 \\ \text { Partial PVD (n) } & 33 \\ \text { Epimacular membrane (n) } & 52 \\ \text { MH (n) } & 25 \\ \text { CFT( } \mu m) & 275.4 \pm 124.6(112-732.5) \\ \text { MNT( } \mu \text { m) } & 413.4 \pm 116.7(206-711) \\ \text { DSM (n) } & 16 \\ \text { Defect of EZ(n) } & 21\end{array}$

$S D$ standard deviation, $D$ diopter, LogMAR logarithm of the minimal angle of resolution, $P V D$ posterior vitreous detachment, $L M H$ lamellar macular hole, EZ ellipsoid zone, CFT central foveal thickness, DSM dome-shaped macula ${ }^{a}$ Eight eyes with SO stage were excluded

\section{Risk factors for progression}

We divided the patients into three groups, progressive, stable and improved, to explore the possible risk factors affecting progression (Table 2). When we determined the influence of the subgroup, subtype and location of retinoschisis on progression, 8 eyes with $\mathrm{S} 0$ at baseline were excluded. The progression rate in eyes with outer schisis most prominently located in the fovea or 


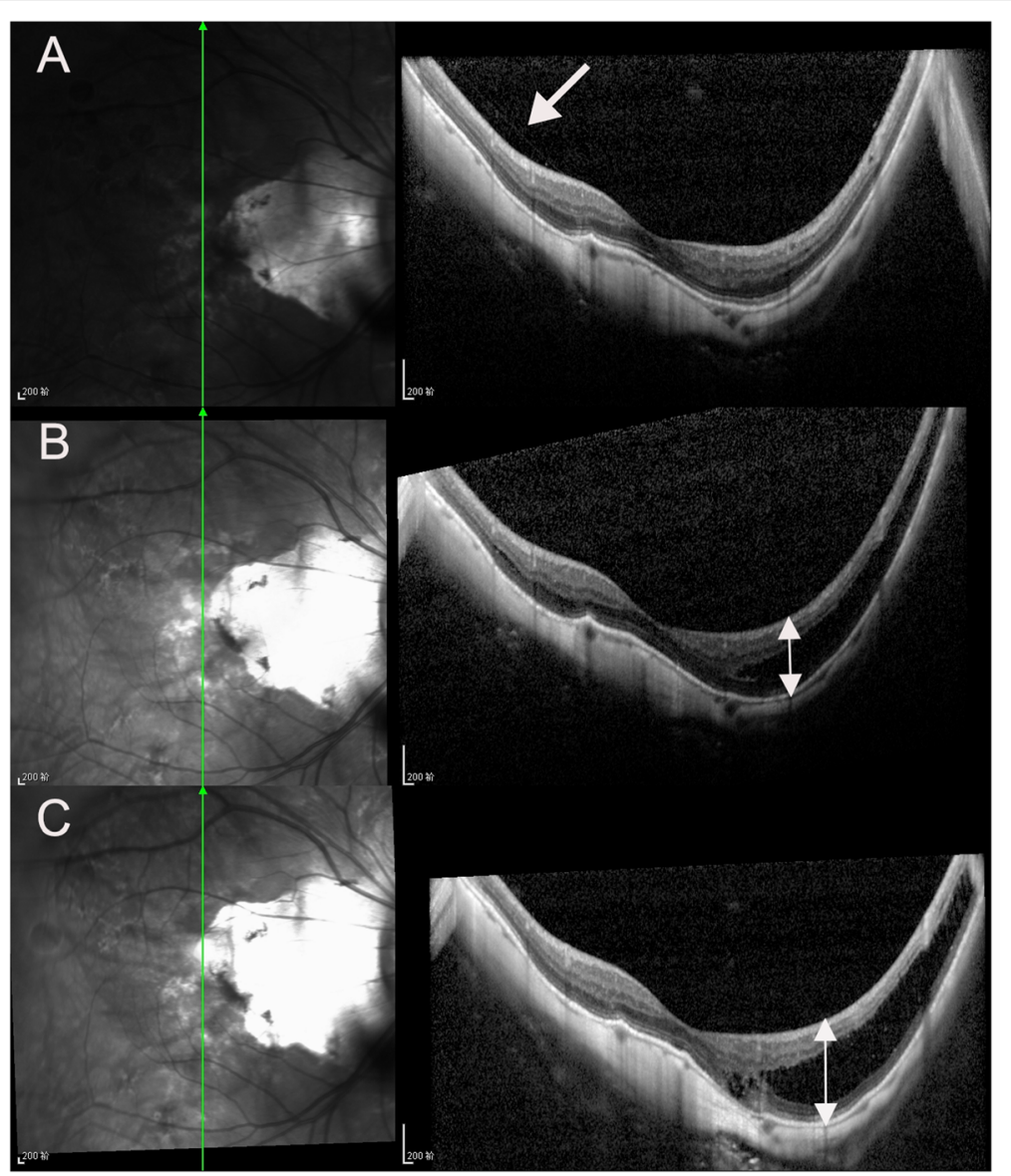

Fig. 1 Infrared fundus and OCT images of the right eye of a 73-year-old man with MTM that progressed during the follow-up period in vertical scan. At baseline, the case had SO MTM with partial posterior vitreous detachment (PVD) (white arrow), the ellipsoid zone (EZ) was intact, and BCVA was 20/63 (A). Tirty-six months later, S3 MTM with outer schisis occurred in the location of posterior staphyloma, and maximum neural thickness (MNT) (white double arrow)was $399 \mu$ m, and BCVA was 20/63 (B). At 68 months after the first visit, the height of schisis increased, and MNT was $525 \mu \mathrm{m}$ (white double arrow), EZ was partially continuous, and BCVA was 20/100 (C)

staphyloma $(51.6 \%)$ was significantly higher than that in eyes with outer schisis located in the vascular arcades $(20.9 \%)(P=0.0011)$. Eyes with partial PVD during the follow-up had a higher proportion of progression $(60.5 \%)$ than those without PVD $(32.9 \%)(P=0.0447)$. In contrast, age, axial length, presence of posterior staphyloma, epimacular membrane, subgroup, subtype of schisis or DSM were not different among the three groups $(P>0.05)$.

\section{Changes in BCVA and predictive factors for visual acuity} LogMAR BCVA at the last follow-up was worse than that at baseline in all patients $(P=0.0372)$, in patients who were older than 65 years, in patients without DSM, and in patients with defects in the EZ $(P=0.0416, P=$ 0.0494 , and $P=0.0130$, respectively) (Table 3 ). In the multiple linear regression analysis, logMAR BCVA $(P<$
$0.0001)$ and a defect in the EZ at baseline $(P<0.0001)$ were significantly correlated with $\log \mathrm{MAR} B C V A$ at the last follow-up (Table 4).

\section{Discussion}

Our study found that $49 / 113$ eyes (43.4\%) with MTM progressed over the 2-year follow-up period, and eyes with retinoschisis prominently located in the fovea or posterior staphyloma were prone to progression. Vitreoretinal traction may be one of the risk factors for progression, and the integrity of the EZ was considered to be a main factor affecting visual prognosis.

The progression rate of MTM or retinoschisis varies, ranging between 11.6 and $68.9 \%$, according to different standards [14, 15]. Gaucher et al. [14] found that $68.9 \%$ (20/29 eyes) of the eyes with foveoschsis progressed with enlargement of retinal cleavage and/or visual decline 


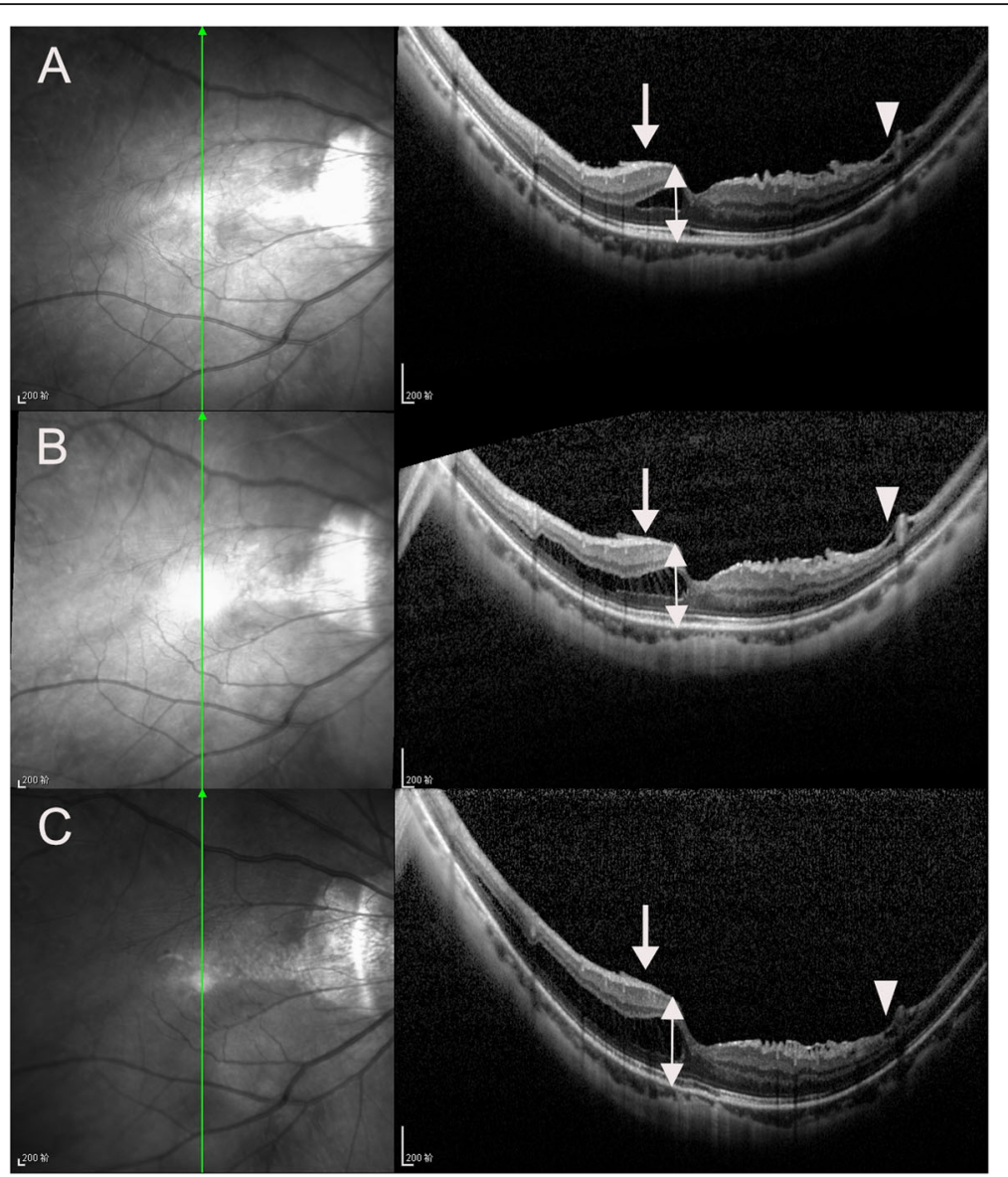

Fig. 2 Infrared fundus and OCT images of the right eye of a 51-year-old woman with MTM that progressed during the follow-up period in vertical scan. At baseline, the case had S2 MTM with outer schisis in fovea, inner schisisin upper vascular arcades (white arrow head) and epimacular membrane (white arrow), MNT (white double arrow) was $361 \mu \mathrm{m}$, and BCVA was 20/32 (A). Twenty-seven months later, S3 MTM occurred, MNT (white double arrow) was $399 \mu \mathrm{m}$, and BCVA decreased to 20/40 (B). At 41 months after the first visit, the extent of schisis enlarged, the height of schisis increased, MNT (white double arrow) was $431 \mu \mathrm{m}$, and BCVA was 20/40. EZ was intact during the follow-up period $(\mathbf{C})$

during a mean follow-up period of 31.2 months. In our retrospective study, 41/105eyes (39.0\%) with MTM progressed based on the criteria of morphological changes of MTM described by Shimada et al. [15], even when 8 S0 eyes were excluded at baseline, which was much higher than that reported by Shimada et al. [15] (11.6\%). The different progression rates may be related to the difference in age, the status of vitreomacular traction, the involved location and area of schisis or the follow-up period in these studies.

Our results showed that eyes with outer schisis prominently located in the fovea or posterior staphyloma had a higher risk of progression than those in which outer schisis located in the vascular areas. The mechanism of retinoschisis is complicated, and the growth of the eyeball is considered an initial factor in the pathogenesis of foveoschisis [19]. Shinohara et al. [20] suggested that posterior staphyloma may act as the main cause of retinoschisis located within the area of the posterior staphyloma by ultrawide-field swept-source OCT, and outer and inner retinoschisis located in vascular arcades may be caused mainly by vitreous adhesion in the blood vessels of the retina and the tractional force of the retinal arterioles $[8,21]$. However, after reaching the loose outer retina through the transmission of intraretinal tissues, the inner traction may become weak, and the inner retina may play a "shock-absorbing"-like role, while outward traction is direct and persistent, which may be the reason why outer retinoschisis at the blood vessel develops more slowly than that in the fovea. In our series, almost all cases of inner schisis were confined to the paravascular area adjacent to the superior or inferior temporal vascular arcades, which may result from tangential traction of the retinal arterioles. The presence of 


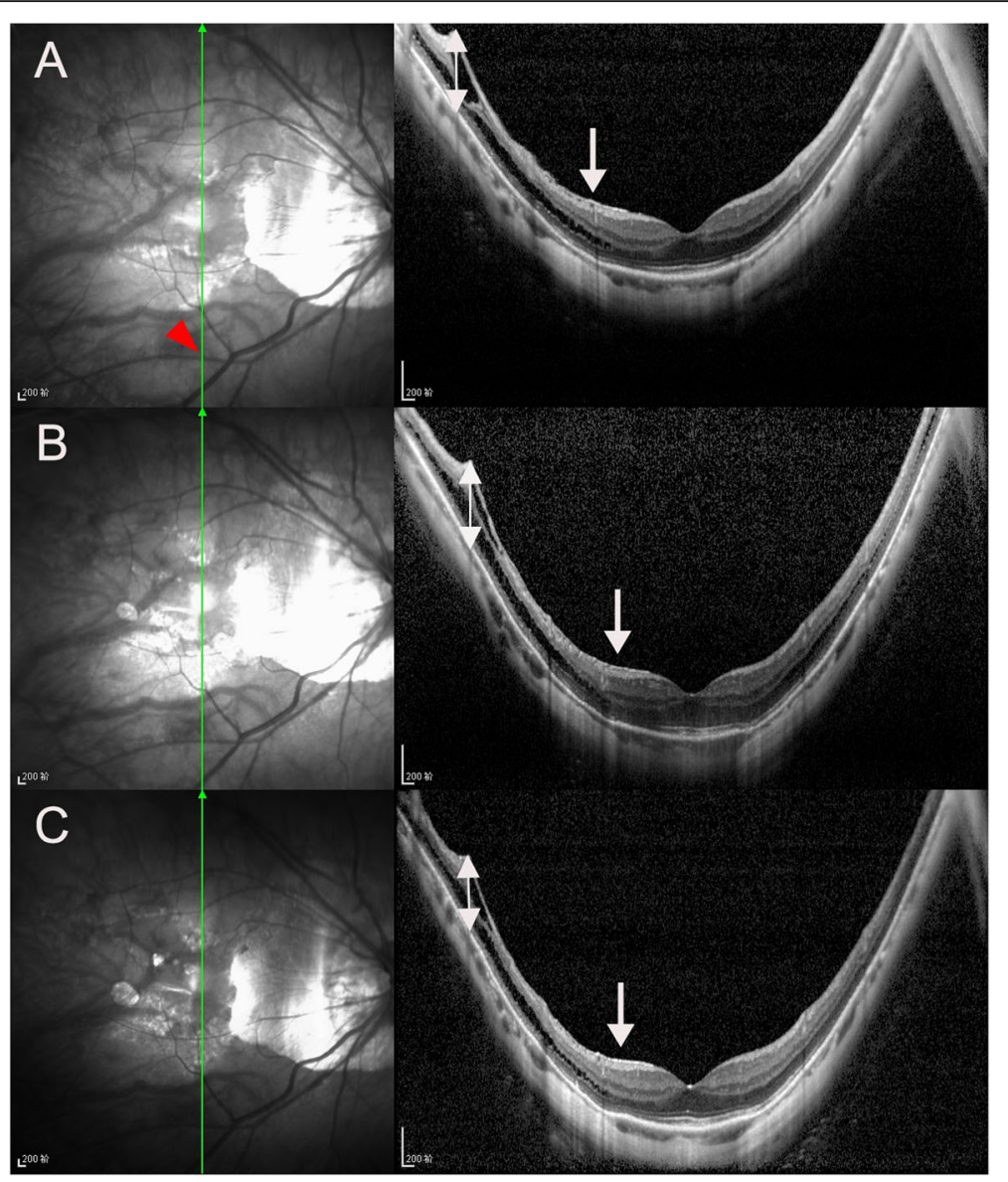

Fig. 3 Infrared fundus and OCT images of the right eye of a 57-year-old man with stable MTM during the follow-up period in vertical scan. At baseline, the case had S3 MTM with outer schisis prominently located in the inferior paravascular area (red arrow head) and inner schisis in the inferior paravascular area, and with epimacular membrane (white arrow), MNT (white double arrow) was 390 um (A). After 53 months, MNT (white double arrow) was $406 \mu \mathrm{m}$ (B). At 67 months after the first visit, MNT (white double arrow) was $405 \mu \mathrm{m}$, EZ was intact and BCVA was 20/ 32 during the follow-up period (C)

inner schisis did not significantly promote the progression of outer schisis in different locations regardless of the presence of ILM detachment, revealing that the retina itself may play a secondary role in the occurrence and development of outer schisis. In this study, MTM with partial PVD during follow-up was more likely to progress. Shinohara et al. [20] also proposed that the posterior vitreous extensively adhered to the retinal surface, exerting persistent inward traction in eyes of retinoschisis, which may contribute to the development of retinoschisis without posterior staphyloma. Additionally, we found that the stage of schisis had no significant influence on the risk of progression. Shimada et al. [15] and Cheng et al. [16] suggested that the status of S4 eyes was unstable and had a high risk for deterioration. Cai et al. [17] also believed that as long as the schisis involved the fovea, regardless of whether it extended to the entire macula, the possibility of progression was relatively high. Therefore, attention should be paid to each stage of MTM, especially in eyes with vitreomacular adhesion. Meanwhile, we observed that eyes in which ILM detachment was disrupted or disappeared did not progress. In foveoschisis, Müller cells and astrocytes proliferate to produce tangential stress to separate the intraretinal tissues [10]. Once ILM detachment is disrupted, tangential stress is released, and the splitting cavity may shrink or even completely disappear. In addition, recent studied have shown that extrafoveal retinoschisis is more common in highly myopic eyes with dome-shaped macula (DSM) than foveal retinoschisis $[22,23]$, and DSM may reduce the traction on the fovea, acting as a macular buckle [18], whereas, in our study, there was no significant difference in the progression rate of MTM between the DSM group and the nonDSM group, and the presence of DSM did not seem to delay the progression of MTM, the possible reason may 


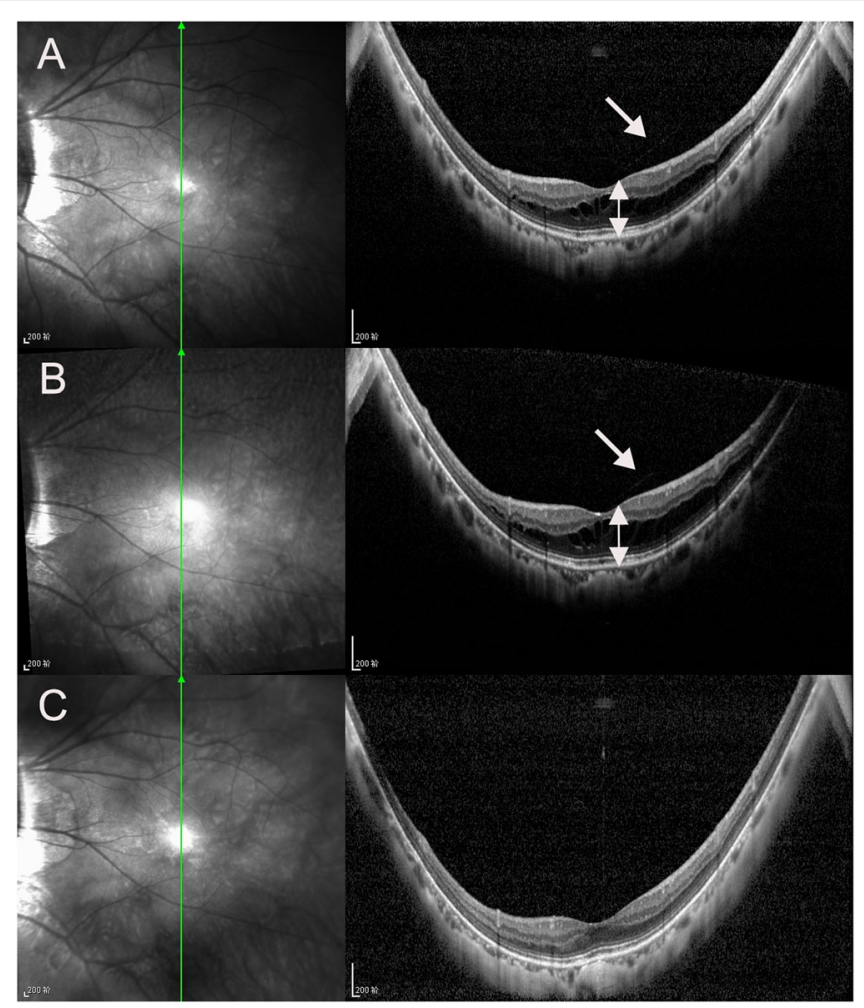

Fig. 4 Infrared fundus and OCT images of the left eye of a 57-year-old woman with MTM for improvement during the follow-up period in vertical scan. At baseline, the case had S3 MTM with outer schisis prominently located in the fovea (also in posterior staphyloma) and partial PVD (white arrow), MNT (white double arrow) were $308 \mu \mathrm{m}$, and BCVA was 20/50 (A). After 10 months, MNT (white double arrow) were $333 \mu \mathrm{m}$, and BCVA was 20/50 (B). At 37 months after the first visit, partial PVD detached from the fovea, the outer schisis disappeared in vertical scan, and BCVA improved to 20/25. EZ was intact during the follow-up period (C)

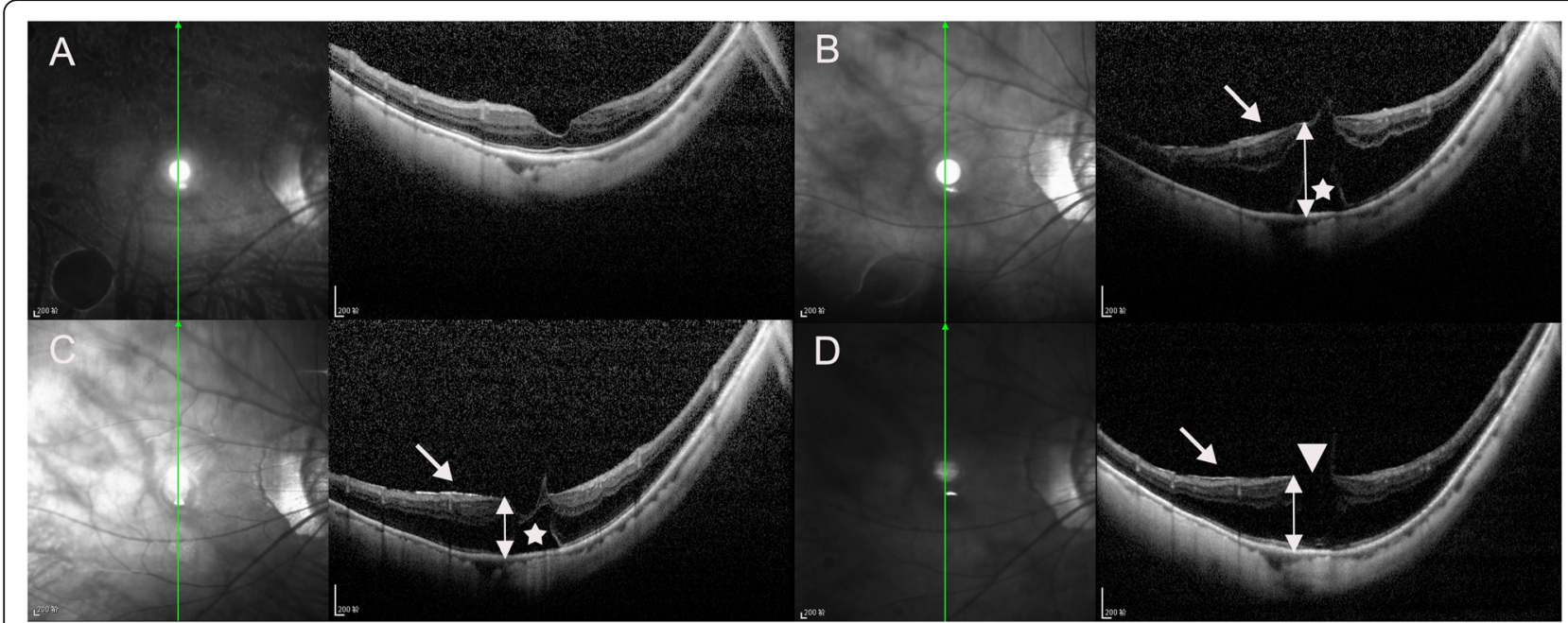

Fig. 5 Infrared fundus and OCT images of the right eye of an 80-year-old woman with MTM for progression during the follow-up period in vertical scan. At baseline, the case had SO MTM, the ellipsoid zone was intact on the fovea, and BCVA was 20/50 (A). After 39 months, S4 retinoschisis with an epimacular membrane (white arrow) was seen and a foveal detachment (FD) (white asterisk) developed, MNT (white double arrow) were $665 \mu \mathrm{m}$ and BCVA declined to 20/200 (B). At 44 months after the first visit, the height of outer retinoschisis decreased, MNT (white double arrow) was $368 \mu \mathrm{m}$, and BCVA was still 20/200 (C). At 67 months after the first visit, the FD resolved spontaneously, a lamellar macular hole (white triangle) developed, the height and of outer retinoschisis increased, MNT (white double arrow) was $496 \mu \mathrm{m}$, while the extent of the outer retinoschisis decreased, and BCVA declined to 20/400 (D) 
Table 2 Factors associated with evolution of myopic traction maculopathy

\begin{tabular}{|c|c|c|c|c|}
\hline Factor & Progressed & Stable & Improved & $P$ value \\
\hline Age (years), mean $\pm S D$ & $64.7 \pm 10.2$ & $66.1 \pm 9.3$ & $62.4 \pm 8.0$ & $0.2267^{*}$ \\
\hline Refractive error (D), mean $\pm S D$ & $-11.8 \pm 4.4$ & $-12.9 \pm 4.4$ & $-13.8 \pm 6.2$ & $0.3233^{*}$ \\
\hline Axial length $(\mathrm{mm})$, mean $\pm \mathrm{SD}$ & $29.3 \pm 1.4$ & $29.3 \pm 2.0$ & $29.3 \pm 2.4$ & $0.9436^{*}$ \\
\hline Posterior staphyloma & & & & $0.8889^{* *}$ \\
\hline Present & 39 & 34 & 15 & \\
\hline Absent & 10 & 11 & 4 & \\
\hline Schisis subgroup $(n)^{a}$ & & & & $0.2916^{* *}$ \\
\hline S1 & 8 & 10 & 6 & \\
\hline S2 & 3 & 5 & 1 & \\
\hline S3 & 20 & 16 & 10 & \\
\hline S4 & 10 & 14 & 2 & \\
\hline Schisis subtype $(n)^{a}$ & & & & $0.4098^{* *}$ \\
\hline Outer schisis & 29 & 26 & 12 & \\
\hline Outer and inner schisis & 12 & 19 & 7 & \\
\hline The most prominent location of outer schisis $(n)^{*}$ & & & & $0.0011^{* *}$ \\
\hline Paravascuslar & 9 & 22 & 12 & \\
\hline Fovea or posterior staphyloma & 32 & 23 & 7 & \\
\hline Epimacular membrane during a follow-up (n) & & & & $0.0636^{* *}$ \\
\hline Present & 27 & 19 & 6 & \\
\hline Absent & 22 & 26 & 13 & \\
\hline Partial PVD during a follow-up(n) & & & & $0.0447^{* *}$ \\
\hline Present & 26 & 10 & 7 & \\
\hline Absent & 23 & 35 & 12 & \\
\hline $\mathrm{LMH}(\mathrm{n})$ & & & & $0.4645^{* *}$ \\
\hline Present & 11 & 12 & 2 & \\
\hline Absent & 38 & 33 & 17 & \\
\hline $\operatorname{DSM}(n)$ & & & & 0.1175 \\
\hline Present & 4 & 8 & 4 & \\
\hline Absent & 45 & 37 & 15 & \\
\hline
\end{tabular}

SD standard deviation, $P V D$ posterior vitreous detachment, $L M H$ lamellar macular hole, DSM dome-shaped macula

${ }^{a}$ Eight eyes with $S 0$ stage were excluded

* Significant difference among group means for age and axial length via one-way analysis of variance $(P<0.05)$

** Significant difference among three groups for the other factors via chi-square test $(P<0.05)$

be that the existence of DSM was not enough to completely offset or resist the inward or tangential traction force acting on the retina or the shape of the posterior staphyloma changed during follow-up period in our cases.

In this study, patients who were older than 65 years, who had not a DSM, and who had EZ defects at the first visit had poor final BCVA, and further multiple linear regression analysis showed that first-visit visual acuity and EZ defects are factors affecting visual prognosis. The interruption or absence of reflection EZ in the outer retina of retinoschisis may represent the abnormal energy metabolism of the elongated mitochondria of the photoreceptors, which means that visual function is impaired. Damage to EZ is not uncommon in patients with retinoschisis. Sayanagi et al. $[24,25]$ reported that the incidence of EZ defects in foveoschisis is between 29 and $38 \%$. Studies on the influencing factors of vision recovery after vitrectomy for foveoschisis have confirmed that the recovery of the EZ has a significant correlation with postoperative vision recovery $[26,27]$. Cheng et al. [16] reported that 6/14 (42.9\%) patients with retinoschisis who had $\geq 2$ lines of vision loss in their natural courses had EZ disruption at the first visit. We speculate that 
Table 3 Comparison of visual acuity at first and last visits with different baseline characteristics

\begin{tabular}{|c|c|c|c|}
\hline \multirow[t]{2}{*}{ Characteristics } & \multirow{2}{*}{$\begin{array}{l}\text { BCVA at First Visit } \\
\text { Mean } \pm S D, \text { logMAR }\end{array}$} & \multirow{2}{*}{$\begin{array}{l}\text { BCVA at Last Visit } \\
\text { Mean } \pm S D, \text { logMAR }\end{array}$} & \multirow[t]{2}{*}{$P$ value* } \\
\hline & & & \\
\hline All patients & $0.42 \pm 0.39$ & $0.58 \pm 0.53$ & 0.0372 \\
\hline \multicolumn{4}{|l|}{ Age } \\
\hline$\geq 65$ years & $0.49 \pm 0.39$ & $0.71 \pm 0.56$ & 0.0416 \\
\hline$<65$ years & $0.35 \pm 0.37$ & $0.45 \pm 0.48$ & 0.2603 \\
\hline \multicolumn{4}{|l|}{ Axial length } \\
\hline$>30 \mathrm{~mm}$ & $0.45 \pm 0.44$ & $0.65 \pm 0.63$ & 0.2740 \\
\hline$<30 \mathrm{~mm}$ & $0.40 \pm 0.36$ & $0.54 \pm 0.48$ & 0.0702 \\
\hline \multicolumn{4}{|l|}{ Posterior staphyloma } \\
\hline Present & $0.44 \pm 0.39$ & $0.63 \pm 0.56$ & 0.0565 \\
\hline Absent & $0.32 \pm 0.35$ & $0.40 \pm 0.40$ & 0.3444 \\
\hline \multicolumn{4}{|l|}{ Lamellar macular hole } \\
\hline Present & $0.72 \pm 0.49$ & $0.99 \pm 0.62$ & 0.1295 \\
\hline Absent & $0.33 \pm 0.30$ & $0.46 \pm 0.45$ & 0.0643 \\
\hline \multicolumn{4}{|l|}{ CFT } \\
\hline$\geq 300 \mu \mathrm{m}$ & $0.58 \pm 0.44$ & $0.79 \pm 0.60$ & 0.1859 \\
\hline$<300 \mu \mathrm{m}$ & $0.34 \pm 0.33$ & $0.48 \pm 0.48$ & 0.0678 \\
\hline \multicolumn{4}{|l|}{ DSM } \\
\hline Present & $0.46 \pm 0.47$ & $0.64 \pm 0.62$ & 0.3931 \\
\hline Absent & $0.41 \pm 0.37$ & $0.57 \pm 0.52$ & 0.0494 \\
\hline \multicolumn{4}{|l|}{ EZ } \\
\hline Intact or partially continuous & $0.34 \pm 0.31$ & $0.44 \pm 0.39$ & 0.1118 \\
\hline Defect & $0.72 \pm 0.52$ & $1.20 \pm 0.64$ & 0.0130 \\
\hline
\end{tabular}

LogMAR logarithm of the minimal angle of resolution, CFT central foveal thickness, DSM dome-shaped macula; EZ ellipsoid zone

*Significant difference between the group means at the first and last visit via Kruskal-Wallis test $(P<0.05)$

some elderly patients had a long course of schisis, and the baseline EZ was not intact or even absent, the photoreceptors were damaged severely over time, which subsequently caused poor vision. Even in pseudophakic eyes, the improvement in their visual function was not satisfactory after cataract surgery. In addition, Zhu and her colleagues [23] proposed that DSM brought better visual acuity after cataract surgery in highly myopic eyes. BCVA of DSM eyes at the final visit was not significantly different from that at the first visit in our study, the

Table 4 Multiple linear regression analysis of baseline characteristics associated with visual acuity at final visit

\begin{tabular}{lcr}
\hline Characteristics & Standardized estimate & $\boldsymbol{P}_{\text {value* }}^{*}$ \\
\hline Age (years) & 0.0445 & 0.4447 \\
Axial Length (mm) & -0.0051 & 0.9296 \\
BCVA (LogMAR) at first visit & 0.6325 & $<0.0001$ \\
Posterior Staphyloma & 0.0523 & 0.3338 \\
LMH & 0.0631 & 0.3729 \\
Schisis Subgroup & 0.0039 & 0.9468 \\
Schisis Subtype & -0.0020 & 0.9705 \\
CFT( $\mu$ m) & 0.0438 & 0.5245 \\
DSM & 0.0304 & 0.5921 \\
Defect of EZ & 0.2718 & $<0.0001$ \\
\hline
\end{tabular}

LogMAR logarithm of the minimal angle of resolution, CFT central foveal thickness, DSM dome-shaped macula, EZ ellipsoid zone *Significant difference via multiple linear regression analysis $(P<0.05)$ 
presence of DSM may reduce the risk of the deterioration of visual acuity, nevertheless, EZ defect was still the leading factor of visual impairment in our study.

Our study has some limitations. First, the occurrence and development of cataracts in some elderly patients who experienced a long follow-up time inevitably affected visual acuity, and an electrophysiological examination may be required as a good supplement to evaluate visual function and prognosis. Second, the exertion of centrifugal vertical and tangential forces may cause an increase in the height and expansion of the extent of retinoschisis, respectively. In future studies, if we can increase the sample size, quantify the extent of the schisis, supplement the criteria for the progression of MTM, and further explore the equivalence of the impact of the expansion of the extent and the increase in height of the schisis on the progression of MTM, it may be of some significance to investigate its pathogenesis.

\section{Conclusions}

In summary, MTM had a high progression rate during the follow-up. MTM progression was related to the location of retinoschisis. Vitreomacular traction may play an important role in the natural course of MTM. Defects in the EZ have a negative influence on visual prognosis, which may provide some clues to the long-term evolution and pathogenesis of MTM.

\section{Abbreviations \\ MTM: Myopic traction maculopathy; EZ: Ellipsoid zone; LMH: Lamellar macular hole; PVD: Posterior vitreous detachment; ILM: Internal limiting membrane; BCVA: Best-corrected visual acuity; Log MAR: Logarithmic minimal angle of resolution; SD-OCT: Spectral domain OCT; CFT: Central foveal thickness; MNT: Maximum neural thickness; SD: Standard deviation}

\section{Acknowledgements}

Not applicable.

\section{Authors' contributions}

Conceived and designed the experiments: SL, QW. Performed the experiments: SL, XW, XC. Analysed the data: SL, TL, CL. Contributed reagents/ materials/analysis tools: BL, YC. Wrote the paper: SL. Discussion of the results and critical review of the manuscript: SL, QW. All authors read and approved the final manuscript.

\section{Funding}

General Program of National Natural Science Foundation of China (81770940).

\section{Availability of data and materials}

The data used and analyzed during the current study are available from the corresponding author on reasonable request. The data supporting our findings can also be found in the Chinese clinical trial registry (http://www. chictr.org.cn/, Registration number: ChiCTR2000038824).

\section{Declarations}

\section{Ethics approval and consent to participate}

The study adhered to the guidelines of the Helsinki Declaration and had the approval of the Ethics Committee of Sixth People's Hospital Affiliated to Shanghai Jiao Tong University, Shanghai, China (Approval NO.: 2020-073). The study was registered in the Chinese clinical trial registry (http://www. chictr.org.cn/, Registration number: ChiCTR2000038824). All patients signed written informed consent for participation.

\section{Consent for publication}

Written informed consents for the publication were obtained from five patients who had the personal and clinical details along with OCT images presented in the article.

\section{Competing interests}

The authors declare that they have no competing interests.

Received: 20 December 2020 Accepted: 30 August 2021

Published online: 25 September 2021

\section{References}

1. Ruiz-Medrano J, Montero JA, Flores-Moreno I, Arias L, García-Layana A, RuizMoreno JM. Myopic maculopathy: current status and proposal for a new classification and grading system (ATN). Prog Retin Eye Res. 2019;69:80-115.

2. Sborgia G, Boscia F, Niro A, Giancipoli E, Amico Ricci GD, Sborgia A, Sborgia L, Recchimurzo N, Romano MR, Addabbo G, Alessio G. Morphologic and functional outcomes of different optical coherence tomography patterns of myopic foveoschisis after vitrectomy and inner limiting membrane peeling. Eye (Lond). 2019:33:1768-75.

3. Lehmann M, Devin F, Rothschild PR, Gaucher D, Morin B, Philippakis E, Massin P, Couturier A, Tadayoni A. Preoperative factors influencing visual recovery after vitrectomy for myopic foveoschisis. Retina. 2019;39:594-600.

4. Hattori K, Kataoka K, Takeuchi J, Ito Y, Terasaki H. Predictive factors of surgical outcomes in vitretomy myopic traction maculopathy. Retina. 2019; 38(Suppl 1):S23-30.

5. Huang Y, Huang W, Danny SC, Ng, Duan A. Risk factors for development of macular hole retinal detachment after pars plana vitrectomy for pathologic myopic foveoschisis. Retina. 2017;37:1049-54.

6. Takano M, Kishi S. Foveal retinoschisis and retinal detachment in severely myopic eyes with posterior staphyloma. Am J Ophthalmol. 1999;128:472-6.

7. Song M, Shen M, Zhou Y, Zheng K, Zhai Y, Xiao M, Wang X, Wang F, Sun X. Observation of vitreous features using enhanced vitreous imaging optical coherence tomography in highly myopic retinoschisis. Retina. 2019;39:173241.

8. Takahashi H, Tanaka N, Shinohara K, Yokoi T, Yoshida T, Uramoto K, OhnoMatsui K. Ultra-widefield optical coherence tomographic imaging of posterior vitreous in eyes with high myopia. Am J Ophthalmol. 2019;206: 102-12.

9. Philippakis E, Couturier A, Gaucher D, Gualino V, Massin P, Gaudric A, Tadayoni R. Posterior vitreous detachment in highly myopic eyes undergoing vitrectomy. Retina. 2016;36:1070-5.

10. Chen L, Wei Y, Zhou X, Zhang Z, Chi W, Gong L, Jiang X, Zhang S. Morphologic, biomechanical, and compositional features of the internal limiting membrane in pathologic myopic foveoschisis. Invest Ophthalmol Vis Sci. 2018:59:5569-78.

11. Yu X, Ma W, Liu B, Li Z, Zhao X, Tanumiharjo S, Chen X, Lyu C, Ao H, Li S, Li $Y$, Lu L. Morphological analysis and quantitative evaluation of myopic maculopathy by three-dimensional magnetic resonance imaging. Eye (Lond). 2018;32:782-7.

12. Li T, Wang X, Zhou Y, Feng T, Xiao M, Wang F, Sun X. Paravascular abnormalities observed by spectral domain optical coherence tomography are risk factors for retinoschisis in eyes with high myopia. Acta Ophthalmol. 2018;96:e515-23

13. Kamal-Salah R, Morillo-Sanchez MJ, Rius-Diaz F, Garcia-Campos JM. Relationship between paravascular abnormalities and foveoschisis in highly myopic patients. Eye (Lond). 2015;29:280-5.

14. Gaucher D, Haouchine B, Tadayoni R, Massin P, Erginay A, Benhamou N, Gaudric A. Long-term follow-up of high myopic foveoschisis: natural course and surgical outcome. Am J Ophthalmol. 2007;143:455-62.

15. Shimada N, Tanaka Y, Tokoro T, Ohno-Matsui K. Natural course of myopic traction maculopathy and factors associated with progression or resolution. Am J Ophthalmol. 2013;156:948-57.

16. Cheng C, Teo K, Tan CS, Lee SY, Loh BK, Wong E, Wong D, Wong TY, Cheung CMG. Myopic retinoschisis in Asians: structural features and determinants of visual acuity and prognostic factors for progression. Retina. 2016;36:717-26. 
17. Cai L, Sun Z, Guo D, Fan Q, Zhu X, Yang J, Lu Y. Long-term outcomes of patients with myopic traction maculopathy after phacoemulsification for incident cataract. Eye (Lond). 2019;33:1423-32.

18. Ellabban AA, Tsujikawa A, Matsumoto A, et al. Three-dimensional tomographic features of dome-shaped macula by swept-source optical coherence tomography. Am J Ophthalmol. 2013;155:320-8 e322.

19. Wu PC, Chen YJ, Chen YH, Chen CH, Shin SJ, Tsai CL, Kuo H-K. Factors associated with foveoschisis and foveal detachment without macular hole in high myopia. Eye (Lond). 2009;23:356-61.

20. Shinohara K, Tanaka N, Jonas JB, Moriyama N, Yoshida M, Ohno-Matsui T. Ultrawide-field OCT to investigate relationships between myopic macular retinoschisis and posterior staphyloma. Ophthalmology. 2018;125:1575-86.

21. Ikuno Y, Gomi F, Tano Y. Potent retinal arteriolar traction as a possible cause of myopic foveoschisis. Am J Ophthalmol. 2005;139:462-7.

22. Fang $\mathrm{D}$, Zhang Z, Wei Y, et al. The morphological relationship between dome-shaped macula and myopic retinoschisis: a cross-sectional study of 409 highly myopic eyes. Invest Ophthalmol Vis Sci. 2020;61:19.

23. Zhu X, He W, Zhang S, et al. Dome-shaped macula: a potential protective factor for visual acuity after cataract surgery in patients with high myopia. Br J Ophthalmol. 2019;103:1566-70.

24. Sayanagi K, Ikuno Y, Soga K, Tano Y. Photoreceptor inner and outer segment defects in myopic foveoschisis. Am J Ophthalmol. 2008;145:90228

25. Sayanagi K, Morimoto Y, Ikuno Y, Tano Y. Spectral-domain optical coherence tomographic findings in myopic foveoschisis. Retina. 2010;30: 623-8.

26. Fujimoto S, Ikuno Y, Nishida K. Postoperative optical coherence tomographic appearance and relation to visual acuity after vitrectomy for myopic foveoschisis. Am J Ophthalmol. 2013;156:968-73.

27. Shin JY, Yu HG. Visual prognosis and spectral-domain optical coherence tomography findings of myopic foveoschisis surgery using 25-gauge transconjunctival sutureless vitrectomy. Retina. 2012;32:486-92.

\section{Publisher's Note}

Springer Nature remains neutral with regard to jurisdictional claims in published maps and institutional affiliations.

Ready to submit your research? Choose BMC and benefit from:

- fast, convenient online submission

- thorough peer review by experienced researchers in your field

- rapid publication on acceptance

- support for research data, including large and complex data types

- gold Open Access which fosters wider collaboration and increased citations

- maximum visibility for your research: over $100 \mathrm{M}$ website views per year

At $\mathrm{BMC}$, research is always in progress.

Learn more biomedcentral.com/submissions 